\title{
Extremity in surgeon volume: Korea may be the place to go if you want to be a decent gastric surgeon
}

\author{
Yasuhiro Kodera ${ }^{1}$
}

Received: 22 February 2015/Accepted: 17 March 2015/Published online: 5 April 2015

(c) The International Gastric Cancer Association and The Japanese Gastric Cancer Association 2015

The relationship between hospital/surgeon volume and various performance indicators is a well-discussed topic in the literature. Various techniques for conventional open surgery have been inevitably and undeniably learned through hands-on training, and superior short- and longterm outcomes can be expected from surgeons who perform a greater number of surgeries per year [1]. Reflecting the importance of a team-oriented approach, hospital volume also has a significant influence on these performance indicators [2].

There is a learning curve in various procedures whereby surgeons need to perform a given procedure a certain number of times before a relevant outcome measure reaches a plateau. The number of cases needed for this may depend on factors such as the complexity of the procedure, how gifted the surgeon is, the quality of the teaching program and the amount of experience the surgeon has had with other related procedures. Several authors consider the learning curve phase to end when performance indicators such as the amount of bleeding, duration of surgery and mortality have decreased to a minimum and then stabilized. However, since the goal of oncological surgery is to cure cancer, the quality of surgery should ultimately be reflected in the long-term outcome. Kim et al. [3] made extensive survival analyses of gastric cancer patients treated by young and upcoming surgeons through the conventional

This comment refers to the article available at doi:10.1007/s10120015-0477-0.

Yasuhiro Kodera

ykodera@med.nagoya-u.ac.jp

1 Department of Gastroenterological Surgery, Nagoya University Graduate School of Medicine, 65 Tsurumai-cho, Showa-ku, Nagoya 466-8550, Japan open approach and observed that 100 patients had to be treated before the oncological outcome became consistent. Indeed, there were significant differences in survival curves among the stage II-IV subsets between patients who received surgery when the surgeon had performed up to 100 gastrectomies and those treated after the surgeon had completed over 100 gastrectomies. While the difference in the stage IV subset may also be attributed to the skill in selecting patients, the differences in the stage II/III subsets might indeed reflect the expertise in the surgical technique. In addition, the duration of surgery decreased incrementally as the surgeon gained experience, while lymph node retrieval increased significantly.

In the well-known British D2 versus D1 randomized trial, a high surgical mortality rate seemed to have hampered the potential survival benefit, if any, of D2 dissection. Related to that trial, Parikh et al. [4] reported the apparent learning curve for the D2 dissection to be 15-25 cases over a period of 18-24 months, but several participating surgeons had little experience in actually performing D2 dissections independently before the trial started. This and the similar results in the Dutch D2 versus D1 trial ultimately led to the concept of centralization: that complex and infrequently performed procedures such as surgery for gastric and esophageal cancer be performed at high-volume centers. In The Netherlands, this concept reportedly led to successful centralization of esophageal cancer patients to hospitals with a minimum of 20 resections annually, and a nonlinear decrease in mortality was observed until the case volume increased to 40-60 esophagectomies a year, where the plateau was reached [5]. In the USA, a hospital with an annual number of $>19$ esophagectomies and $>21$ gastrectomies [1] and a surgeon who has performed $>6$ esophagectomies [2] was ranked as high volume in pioneering studies on the hospital/surgeon 
volume. These findings indicate that the West Europeans and North Americans are more or less in the same league regarding the case volume of upper gastrointestinal tract cancers. Unfortunately, in these regions where the incidence of gastric cancer has declined, performing 100 gastrectomies while in training is unrealistic for surgeons.

In addition to the sheer number, another important parameter indicating the intensity of surgical training is the time needed for a surgeon to experience a given number of the surgical procedures. Only surgeons who have performed at least $150 \mathrm{R}_{0}$ resections in 5 years are eligible according to the study by Kim et al. This criterion also seems rather unrealistic, not only in the West but also in the average institutions in Japan. However, readers of the article who consider such numbers to be ridiculous need to be informed that there are several super high-volume hospitals in South Korea where well over 1,000 gastrectomies are performed annually. This huge number of operations is usually accomplished by a small number of staff surgeons assisted by surgical residents, leading to an extremely high surgeon volume, as evidenced in the Kim study. Amazingly, these hospitals are not only specialized in gastric cancer, but are also rated as high volume for other types of cancer. Such a feat is only possible with excellent hospital infrastructures and management. The hospitals I visited were equipped with operating theaters with their own canteens and cafés where staff surgeons spend 2 whole days a week performing 3-5 gastrectomies per day: this only adds up to 300 a year, taking into account the holidays and absences for other reasons. Hospital beds and recovery rooms need to be managed extremely efficiently: low morbidity and short hospital stays are prerequisites and are made possible by expertise, revealed by the association between surgeon/hospital volume and outcome.

The weakness of the article by Kim et al. is that it deals only with open surgery, ignoring the influence of the emerging number of gastrectomies currently performed using the laparoscopic approach. Indeed, an increasing number of gastrectomies are being performed laparoscopically in Eastern Asia, usually for early-stage cancers. While this trend may deprive young surgeons of opportunities to perform less demanding gastrectomies using the open approach, they will instead have chances to experience laparoscopic surgery with clear and magnified views to better understand the anatomy. Nevertheless, data on the experience each surgeon may have had with the novel approach have not been presented and discussed. The learning curves for open and laparoscopic surgery actually influence each other as a surgeon is trained for both approaches simultaneously, and the case volume needed to be proficient in both approaches will have to be evaluated and discussed as a new topic in another study. Actually, many articles have investigated the learning curve in laparoscopic and even robot-assisted gastrectomy, but these only tell us about surgeons who acquired sufficient expertise in open surgery and then worked hard to adapt to the new approach.

Depressingly, a study in this setting from another highvolume hospital in Korea postulates that a further 100 cases are needed for an established surgeon to reach a plateau in performing total gastrectomy laparoscopically [6]. If this is indeed true, not only surgeons in the West but even those in Japan might need to think again if they are beginning to introduce this challenging procedure. However, I am not quite convinced that it is only the sheer number that matters. In the Japanese institutions where the case volume is more limited, the process of scrutinizing a video of laparoscopic surgery on the same day it is performed is considered a compulsory exercise for a surgical resident given the chance to operate [7]. In addition, surgeons in training should have numerous opportunities to assist the teaching surgeons and afterwards review videos of exemplarily conducted surgery. In the modern era of laparoscopic surgery, videos could assist serious and hardworking surgical apprentices and compensate to a certain extent for the limited number of surgeries actually performed.

For many years, the Japanese media has been obsessed with annually compiling a "Michelin guide" that ranks hospitals from 1 to somewhere around 100 according to the annual number of surgeries performed for each disease category instead of the food, décor or service. The people in the media seem to enjoy playing with the numbers and seem not to notice that the quality of surgery will eventually reach a plateau at some point in the hospital volume. From another angle, it is not easy for a surgeon who performs 300 gastrectomies annually to remember a patient by the voice or name. Not a few Japanese patients seem to expect (or have the illusion) that a surgeon in charge can recognize the clinical stage and potential problems as soon as a patient identifies themselves on the telephone 3 years after surgery. I have received several phone calls that start with "Hello, I'm Suzuki" and immediately move on to the clinical details, seeking advice. I cannot imagine what is going to happen if Mr. Kim or Mrs. Lee acts in the same way in South Korea (N.B., Kim and Lee are the most common surnames in Korea, just as Suzuki is in Japan). If this much service is expected, patients are advised to visit a moderate-volume hospital that nevertheless provides decent surgical treatment. Although only 120 gastrectomies are performed annually at my institution, I have not lost hope that we are still delivering good surgical procedures and educating young surgeons appropriately. Even if somewhat short of ammunition, Japan needs to compete against South Korea, a country that was once a disciple but is now a king in the treatment of gastric cancer. 


\section{References}

1. Birkmeyer JD, Stukel TA, Siewers AE, Goodney PP, Wennberg DE, Lucas FL. Surgeon volume and operative mortality in the United States. N Engl J Med. 2003;349:2117-27.

2. Birkmeyer JD, Siewers AE, Finlayson EVA, Stukel TA, Lucas FL, Batista I, et al. Hospital volume and surgical mortality in the United States. N Engl J Med. 2002;346:1128-37.

3. Kim CY, Nam B-H, Cho GS, Ryu KW, Hyung WJ, Kim MC, et al. Learning curve for gastric cancer surgery based on actual survival. Gastric Cancer. 2015 (in press).

4. Henneman D, Dikken JL, Putter H, Lemmens VE, Van der Geest LG, van Hillegersberg T, et al. Centralization of esophagectomy: how far should we go? Ann Surg Oncol. 2014;21:4068-74.
5. Parikh D, Johnson M, Chagla L, Lowe D, McCulloch P. D2 gastrectomy: lessons from a prospective audit of the learning curve. Br J Surg. 1996;83:1595-9.

6. Jung DH, Son SY, Park YS, Shin DJ, Ahn HS, Ahn SH et al. The learning curve associated with laparoscopic total gastrectomy. Gastric Cancer. 2014 (Epub ahead of print).

7. Yoshikawa T, Cho H, Rino Y, Yamamoto Y, Kimura M, Fukunaga $\mathrm{T}$, et al. A prospective feasibility and safety study of laparoscopyassisted distal gastrectomy for clinical stage I gastric cancer initiated by surgeons with much experience of open gastrectomy and laparoscopic surgery. Gastric Cancer. 2013;16:126-32. 